\title{
Gallifantes. Lectura crítica de estudios híbridos
}

Molina Arias M.

Hospital Infantil Universitario La Paz. Madrid.

\section{Resumen}

Para aquellos casos en los que los estudios observacionales clásicos no se ajustan bien a las necesidades del investigador, se han diseñado una serie de estudios híbridos que aprovechan las ventajas de los estudios convencionales y se adaptan mejor a otras situaciones. Hablaremos de los estudios de casos y controles anidados en una cohorte, de los estudios de cohorte y caso y, finalmente, de los estudios cruzados o de casos y autocontroles.

\section{Introducción}

Para aquellos casos en los que los estudios observacionales clásicos no se ajustan bien a las necesidades del investigador, se han diseñado una serie de estudios híbridos que aprovechan las ventajas de los estudios convencionales y se adaptan mejor a otras situaciones. Hablaremos de los estudios de casos y controles anidados en una cohorte, de los estudios de cohorte y caso y, finalmente, de los estudios cruzados o de casos y autocontroles.

La mezcla irreal de diferentes partes de animales ha sido una manía de los llamados seres humanos desde tiempos inmemoriales. El caso más emblemático es el de Quimera (que da nombre a toda la familia de mezclas de animales diferentes). Este ser mitológico, hija de Tifón y de la víbora Echidna, tenía cabeza de león, cuerpo de cabra y cola de dragón, lo que le permitía respirar llamas y acojonar a todo el que pasaba por su camino. Claro que no le sirvió de nada cuando Belloforontes, montado sobre Pegaso (otro bicho raro, un caballo con alas) se empeñó en atravesarla con su lanza de plomo. Ya veis, en su fuerza estuvo su perdición: el fuego derritió la punta de la lanza dentro de esta rara criatura, lo que produjo su muerte.

Además de Quimera hay muchos más, fruto de la imaginación humana. Por nombrar algunos, podemos recordar a los unicornios (estos tuvieron peor suerte que Pegaso, en lugar de alas tenían cuernos, uno cada animal), los basiliscos (una especie de gallo serpiente de bastante mal carácter), los grifos (cuerpo de león y lo demás de águila) y todos aquellos en los que parte de la mezcla es humana, como las mantícoras (cabeza de hombre y cuerpo de león), los centauros, el Minotauro, Medusa (con sus serpientes en lugar de cabellos), las sirenas...

De todas formas, de entre todos los seres de este zoo imaginario, yo me quedo con el gallifante. Este era una mezcla de gallo y elefante que se utilizaba en la tele para premiar el ingenio de los niños que asistían a un popular concurso. Los milenials no tendréis ni idea de lo que estoy hablando, pero seguro que los que 
crecisteis en los 80 sí sabéis a qué me refiero.

Y todo esto me ha venido a la cabeza cuando estaba reflexionando sobre la cantidad de quimeras que también existen entre los posibles tipos de diseños de estudios científicos, especialmente entre los estudios observacionales. Vamos a conocer un poco tres de estos gallifantes de la epidemiología: los estudios de casos y controles anidados en una cohorte y los estudios de cohorte y caso, para terminar con otro espécimen particular, los estudios cruzados o de casos y autocontroles.

Dentro de estos estudios observacionales, todos conocemos los clásicos estudios de cohortes y los de casos y controles, los más utilizados.

En un estudio de cohortes, un grupo o cohorte es sometido a una exposición y se le sigue a lo largo del tiempo para comparar la frecuencia de aparición del efecto en comparación con una cohorte no expuesta, que actúa como control. Estos estudios suelen ser de direccionalidad anterógrada, por lo que permiten medir la incidencia de la enfermedad y calcular el riesgo relativo entre los dos grupos. Por su parte, en un estudio de casos y controles se parte de dos grupos de población, uno de los cuales presenta el efecto o enfermedad en estudio y se compara su exposición a un factor determinado respecto al grupo que no tiene la enfermedad y que actúa como control. Al ser de direccionalidad retrógrada y seleccionar directamente los casos de enfermedad, no es posible calcular directamente la densidad de incidencia $\mathrm{y}$, por tanto, los riesgos relativos entre los dos grupos, por lo que la medida de asociación típica de los estudios de casos y controles es la odds ratio.
El estudio de cohortes es el más sólido de los dos desde el punto de vista metodológico. El problema es que suelen requerir largos periodos de seguimiento y grandes cohortes, sobre todo cuando la frecuencia de la enfermedad estudiada es baja, lo que lleva a la necesidad de manejar todas las covariables de toda esta gran cohorte, lo que aumenta los costes del estudio.

Pues bien, para estos casos en los que ni los casos y controles ni las cohortes se ajustan bien a las necesidades del investigador, los epidemiólogos han inventado una serie de diseños que están a caballo entre los dos y pueden mitigar sus defectos. Estos diseños híbridos son los estudios de casos y controles anidados en una cohorte y los estudios de cohorte y caso a los que ya nos hemos referido.

En otro orden de cosas, en los estudios observacionales clásicos el punto clave está en la selección de los controles, que tienen que ser representativos del nivel de exposición al factor de riesgo evaluado en la población de la que proceden los casos. Una selección adecuada de los controles se hace todavía más difícil cuando el efecto es de producción brusca. Por ejemplo, si queremos saber si una comida copiosa aumenta el riesgo de infarto tendríamos una gran dificultad para recoger controles representativos de la población, ya que los factores de riesgo pueden actuar instantes antes del evento.

Para evitar estas dificultades se aplicó el principio de "yo me guiso, yo me lo como" y se diseñó el tercer tipo de quimera que hemos mencionado, en el que cada participante actúa, a la vez, como su propio control. Son los estudios cruzados, también llamados de casos y autocontroles. 
Vamos a ver estos bichos raros, empezando con los estudios de casos y controles anidados.

Supongamos que hemos hecho un estudio en el que hemos utilizado una cohorte con muchos participantes. Pues bien, podemos reutilizarla en un estudio de casos y controles anidados. Tomamos la cohorte y la seguimos a lo largo del tiempo, seleccionando como casos aquellos sujetos que van desarrollando la enfermedad y asignándoles como controles sujetos de la misma cohorte que todavía no la han presentado (aunque pueden hacerlo más tarde). Así, casos y controles provienen de la misma cohorte. Es conveniente emparejarlos teniendo en cuenta variables confusoras y dependientes del tiempo como, por ejemplo, los años que llevan incluidos en la cohorte. De esta forma, un mismo sujeto puede actuar como control en varias ocasiones $y$ terminar como caso en otra, lo que habrá que tener en cuenta a la hora del análisis estadístico de los estudios. Como esto parece un poco confuso, os muestro un esquema de este tipo de estudios en la figura 1.

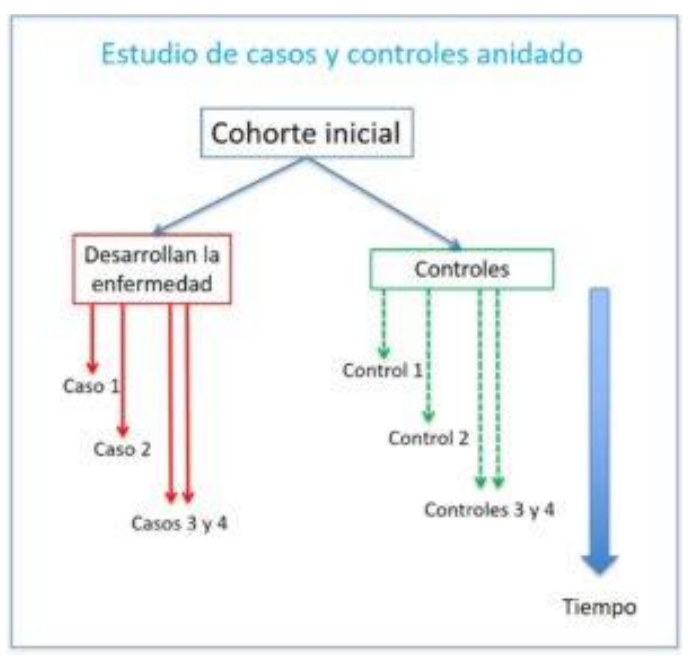

Como vamos viendo cómo surgen los casos, vamos haciendo un muestreo por densidad de incidencia, lo que nos va a permitir estimar riesgos relativos. Esta es una diferencia importante con los estudios de casos y controles convencionales, en los que suele calcularse una odds ratio, que solo puede asimilarse al riesgo relativo cuando la frecuencia del efecto es muy baja.

Otra diferencia es que toda la información sobre la cohorte se recoge al inicio del estudio, por lo que hay menos riesgo de que se produzcan los sesgos de información clásicos de los estudios de casos y controles, de naturaleza habitualmente retrospectiva.

El otro tipo de diseño observacional híbrido que vamos a tratar es el de los estudios de caso y cohorte. Aquí también partimos de una gran cohorte inicial, de la que seleccionamos una subcohorte más manejable que se utilizará como grupo de comparación. Así, vamos viendo qué individuos de la cohorte inicial desarrollan la enfermedad y los comparamos con la subcohorte (con independencia de que pertenezcan o no a la subcohorte). Podéis ver el esquema de un estudio de caso y cohorte en la figura 2 .

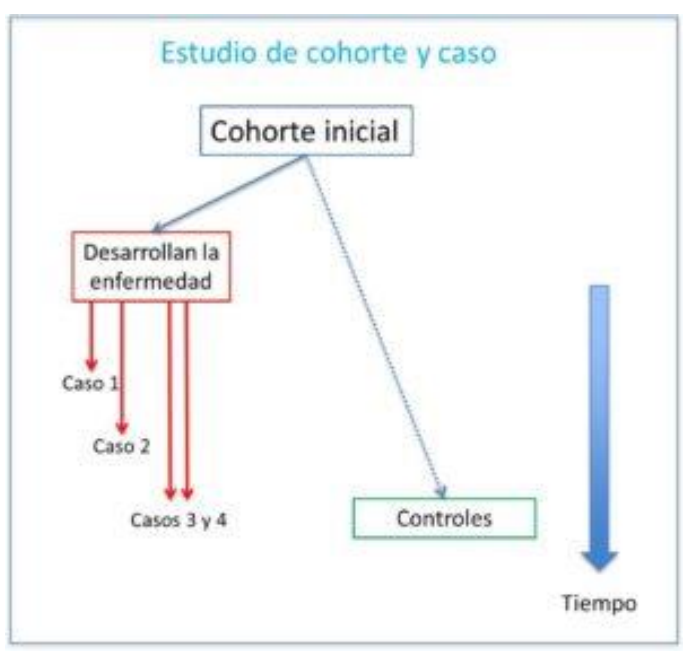

Al igual que en el ejemplo anterior, al elegir los casos a lo largo del tiempo podemos estimar la densidad de incidencia en casos y no casos, calculando a partir de ellas el riesgo relativo. Como podemos imaginar, este 
diseño es más económico que los estudios convencionales porque disminuye mucho el volumen de información de los sujetos sanos que hay que manejar, sin perder eficiencia a la hora de estudiar enfermedades raras. El problema que surge es que la subcohorte tiene una sobrerrepresentación de casos, por lo que el análisis de los resultados no puede hacerse como en las cohortes tradicionales, sino que tiene su metodología propia, bastante más complicada.

Para resumir un poco lo dicho hasta ahora, diremos que el estudio de casos y controles anidado se parece más al de casos y controles clásico, mientras que el de cohorte y caso se parece más al estudio de cohortes convencional. La diferencia fundamental entre los dos es que en el estudio anidado el muestreo de los controles se hace por densidad de incidencia y mediante emparejamiento, por lo que hay que esperar a que se hayan producido todos los casos para seleccionar toda la población de referencia. Esto no es así en el de caso cohorte, mucho más sencillo, en el que la población de referencia se selecciona al comienzo del estudio.

Para acabar con estos estudios híbridos, diremos algunas cosas sobre los estudios cruzados. Estos se centran en el momento en que se produce el evento y tratan de ver si se ha producido algo inusual que lo haya favorecido, comparando las exposiciones de momentos inmediatos al evento con otros anteriores que sirven de control. Por tanto, comparamos momentos casos con momentos controles, actuando cada individuo como su propio control.

Para que el estudio tenga validez desde el punto de vista metodológico, los autores tienen que describir con claridad una serie de periodos de tiempo característicos. El primero es el periodo de inducción, que es el tiempo de retraso que se produce desde el inicio de la exposición hasta la producción del efecto.

El segundo es el periodo de efecto, que es el intervalo durante el cual la exposición puede desencadenar el efecto. Por último, el periodo de riesgo sería la suma de los dos periodos previos, desde que se produce la exposición hasta el inicio del evento.

El periodo de inducción suele ser muy breve la mayor parte de las veces, así que periodo de riesgo y efecto suelen ser equivalentes. En la figura 3 se muestra la relación entre los tres periodos para que la entendáis mejor.

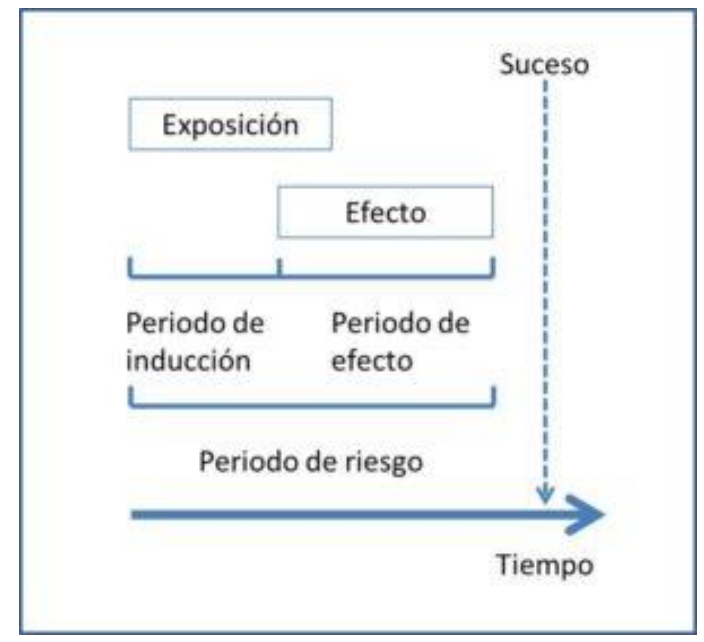

Es fundamental que estos tres periodos se especifiquen con claridad, ya que una mala estimación del periodo de efecto, tanto por exceso como por defecto, produce una dilución del efecto de la exposición y dificulta su detección.

Algunos de vosotros me diréis que estos estudios son similares a otros estudios con autocontroles, como el de casos y controles emparejados. La diferencia es que en este último se eligen uno o varios controles similares por cada caso, mientras que en el autocontrolado cada uno es su propio control. También se parecen un poco a los ensayos clínicos cruzados, en el que todos los 
participantes son sometidos a la intervención y al control, pero éstos son estudios experimentales en los que el investigador interviene en la producción de la exposición, mientras que los autocontrolados son estudios observacionales.

En lo que sí se parecen a los casos y controles emparejados es en el análisis estadístico, solo que aquí se analizan momentos caso y momentos control. De esta forma, lo habitual es utilizar modelos de regresión logística condicional, siendo la medida de asociación más habitual la odds ratio.

Como veis, los estudios híbridos son toda una nueva familia que amenaza con ir creciendo en número $\mathrm{y}$ complejidad. Hasta donde yo sé, no existen listas de verificación para realizar la lectura crítica de estos tipos de diseños, así que tendremos que aplicar de forma juiciosa los principios que aplicamos al analizar los estudios observacionales clásicos, teniendo en cuenta, además, las particularidades de cada tipo de estudio.

Para ello, seguiremos nuestros tres pilares: validez, importancia $\mathrm{y}$ aplicabilidad.

En el apartado de VALIDEZ valoraremos el rigor metodológico con el que está hecho el estudio. Comprobaremos que hay una definición clara de la población de estudio, de la exposición y del efecto. Si utilizamos una cohorte de referencia, esta deberá ser representativa de la población y deberá seguirse de forma completa. Por su parte, los casos serán representativos de la población de casos de la que proceden y los controles de una población con un nivel de exposición representativo de la población de casos.

La medición de la exposición y del efecto deberá hacerse de manera ciega, siendo independiente la medición del efecto del conocimiento del nivel de exposición. Además, analizaremos si se ha prestado atención a la relación temporal de los acontecimientos entre exposición y efecto y si hubo relación entre el nivel de exposición y el grado de efecto. Finalmente, el análisis estadístico deberá ser el correcto, teniendo en cuenta el control de los posibles factores de confusión. Esta parte puede ser complicada por la complejidad de los estudios estadísticos que suelen precisar este tipo de diseños.

Además, como ya hemos comentado, si estamos ante un estudio cruzado habrá que asegurarse de que ha habido una correcta definición de los tres periodos, en especial del periodo de efecto, cuya imprecisión puede afectar en mayor grado la conclusión del trabajo.

A continuación, pasaremos a valorar la IMPORTANCIA de los resultados, así como su precisión, medida por sus intervalos de confianza. Buscaremos las medidas de impacto calculadas por los autores del trabajo y, en caso de que no las proporcionen, trataremos de calcularlas nosotros mismos. Por último, compararemos los resultados con otros previamente existentes en la literatura para ver si son concordantes con el conocimiento existente y qué aportan de nuevo.

Acabaremos la lectura crítica valorando la APLICABILIDAD de los resultados. Pensaremos si los participantes se pueden asimilar a nuestros pacientes $\mathrm{y}$ si las conclusiones son aplicables a nuestro medio.

$\mathrm{Y}$ aquí vamos a ir terminando esta entrada. Hemos visto toda una nueva gama de estudios híbridos que combinan las ventajas de dos estudios observacionales para adaptarse mejor a situaciones en las que los estudios clásicos son más difíciles de aplicar. El 
inconveniente de estos estudios, como ya hemos comentado, es que el análisis es un poco más complicado que el de los estudios convencionales, ya que no es suficiente con el análisis crudo de los resultados, sino que hay que ajustar por la posibilidad de que un participante pueda actuar como control y caso (en los estudios anidados) y por la sobrerrepresentación de los casos en la subcohorte (en los de caso y cohorte).

Acabo ya comentando que todo lo que hemos dicho sobre los estudios cruzados se refiere a los denominados estudios cruzados unidireccionales, en los que existe una relación temporal muy puntual entre exposición y efecto. Para los casos en que la exposición es más mantenida pueden emplearse otros tipos de estudios cruzados llamados bidireccionales, en los que se seleccionan periodos de control anteriores y posteriores al efecto. Pero esa es otra historia...

\section{Bibliografía}

- Delgado Rodríguez M, Sillero M. Revisión: diseños híbridos de estudios de cohortes y de estudios de casos y controles. Gac Sanit.1995;9:42-52. (ㅂMTML)

- Onland-Moret NC, van der A DL, van der Schouw YT, Buschers W, Elias SG, van Gils $\mathrm{CH}$, et al. Analysis of case-cohort data: a comparison of different methods. J Clin Epidemiol.2007;60:350-5. (․ㅏbMed)

- Maclure M. The case-crossover design: a method for studying transient effects on the risk of acute events. Am J Epidemiol.1991;133:14453. (PubMed) (PDF)

- Carracedo Martínez E, Tobías A, Saez M, Taracido M, Figueiras A. Fundamentos y aplicaciones. (HTML)

\section{Correspondencia al autor}

Manuel Molina Arias

mma1961@gmail.com

Servicio de Gastroenterología.

Hospital Infantil Universitario La Paz.

Madrid. España.

Aceptado para blog en marzo de 2019. 